\title{
Data Envelopment Analysis of Different Climate Policy Scenarios
}

\section{by}

\section{Valentina Bosetti* and Barbara Buchner**}

* Fondazione Eni Enrico Mattei, C.so Magenta, 6320123 Milano, Italy. Tel: +39 02 52036983; Fax: +39 02 52036946, E-mail: valentina.bosetti@feem.it

** Fondazione Eni Enrico Mattei, Campo S. M. Formosa, Castello 5252, 30122 Venezia, Italy. Present address: International Energy Agency; 9, rue de la Fédération, 75739 Paris Cedex 15, France. Tel: +33 (0)1 4057 6687, Fax: +33 (0)1 4057 6739; E-mail: barbara.buchner@iea.org

\section{Abstract}

Recent developments in the political, scientific and economic debate on climate change suggest that it is of critical importance to develop new approaches able to compare policy scenarios while also taking their performance in terms of the sustainability paradigm into account. This paper discusses a quantitative methodology to assess the relative performance of different climate policy scenarios when accounting for their long-term economic, social and environmental impacts. The proposed procedure is based on Data Envelopment Analysis, here employed in evaluating relative efficiency of ten global climate scenarios. The methodology provides a promising comparison framework; it can be seen as a way of setting some basic guidelines to frame further debates and negotiations and can be flexibly adopted and modified by decision makers to obtain information relevant for policy design. In the present paper, we find that the "do nothing" strategy is inevitably dominated either by moderate or by stringent policies, depending on how much the policy-maker values environmental and social impacts in addition to policy costs. More generally, scenarios that would not pass the economic efficiency test might reveal a good performance in terms of sustainability notwithstanding their relatively high costs.

Keywords: Climate Policy, Valuation, Data Envelopment Analysis, Sustainability

JEL Classification: H41, Q51, Q54, C61

This paper is part of the research work being carried out by the Climate Change Modelling and Policy Unit at Fondazione Eni Enrico Mattei. The authors are grateful to Christoph Böhringer, Carlo Carraro, 


Marzio Galeotti and Henry Jacoby for helpful suggestions and remarks. Financial support from the EU
funded TranSust.Scan project is gratefully acknowledged. The usual disclaimer applies.
1. Introduction

During the last few decades, climate change has evolved as one of the major threats to the earth's sustainability. The political response in the form of the United Nations Framework Convention on Climate Change (UNFCCC, 1997) and more specifically the Kyoto Protocol have started a process towards a new policy architecture better able to cope with the complexities of climate change. However, recent negotiations and developments suggest that it might be difficult to achieve a single global agreement through the usual top -down way of designing policy architectures, and that regional or subglobal agreements are more likely to emerge ${ }^{1}$. The usual way of planning climate policy has led to a certain deadlock in negotiations, which has consequently induced the search for more successful policy architectures to cope with the ever-increasing emissions. Indeed, even though the Kyoto Protocol came into force in February 2005, its environmental effectiveness is very low due to the lack of participation of several key countries, as in particular the world's largest producer of GHG emissions, the US, and large developing countries as China and India. As a consequence, general consensus has emerged that the Kyoto Protocol represents only a first step towards the broader aim of minimising the danger of climate change. ${ }^{2}$ This has stimulated detailed discussions on potential climate policy scenarios and a number of different approaches have been applied in order to analyse the possible future of climate policy. In this context, the recent experiences in the politics and economics of climate change control have given an indication of how important it is to be accurate in measuring the efficiency of efforts towards climate control in order to increase the feasibility of a possible policy scenario.

\footnotetext{
${ }^{1}$ Indeed, due to free-riding incentives and strong economic and environmental asymmetries, it is unlikely that an international climate agreement will be signed by a large number of countries Carraro and Siniscalco, 1993; Botteon and Carraro, 1997), unless its goals are not significantly different from those of a non-cooperative, business-as-usual, domestic policy Barrett, 1994) Therefore, more recently the idea of a global bottom-up climate agreement has been proposed (Cf. Carraro, 1998, 1999; Buchner and Carraro, 2007; Egenhofer et al., 2001; Stewart and Wiener, 2003). The basic idea is to adopt a bottom-up, country-driven approach to defining national commitments, instead of a top-down, international negotiation of national emission targets.

${ }^{2}$ Actually, in July 2005 six nations led by the US and Australia unveiled a complementary pact to the Kyoto Protocol, aimed to fight global warming. The Asia-Pacific Partnership on Clean Development and Climate constitutes a voluntary, technology-based initiative to reduce greenhouse gas emissions without legally binding emissions targets, whose main idea is to develop new technologies and deploy these in developing countries. Notwithstanding the characteristic of being voluntary, this agreement could be interpreted as a further step into the direction of a more comprehensive climate policy. For more information on this pact - signed by the US, Australia, Japan, China, India and South Korea - see http://www.ap6.gov.au/
} 
Yet, given the difficulty of measuring climate policies in a satisfactory way, no strategy that can satisfy all the needs of all countries has yet been identified. This problem is becoming more pressing because of the increasing urgency to improve the credibility of climate policy in general. In order to move forward in climate negotiations, countries need to have a better way of evaluating efforts at their disposal. The paper aims at contributing to this objective.

Two particular reasons stress the importance of such an evaluation tool. First, countries currently outside the int ernational climate architecture need to have instruments to evaluate their next steps in climate policy in order to justify their strategies both domestically and internationally. Second, negotiations on a Post-2012 phase have started given that the Kyoto Protocol contains commitments only through 2012. At the climate talks in Montreal in 2005, a new round of climate talks has been initiated both under the UNFCCC and under the Kyoto Protocol, focusing on the future of the international climate effort. The general stalemate in the Kyoto negotiations suggests that all countries would benefit from a new approach to looking at climate change measures. Above all, focussing exclusively on emissions or emission concentrations or temperature appears to be too narrow ${ }^{4}$. By moving beyond this perspective, the efficiency of climate change control can be evaluated more comprehensively. In particular, given the international commitment towards sustainable development as the overall guideline for all areas of policy making ${ }^{5}$, measurements of efficiency better able to account for the three dimensions of sustainability i.e., the economic, social and environmental aspects - are essential if climate-energy policy is to be more effective and successful.

Attempts to compare and measure climate policies are not new. The climate policy scenarios embedded in most of the existing policy proposals usually represent mitigation scenarios that are defined through a description and a quantified projection of how GHG emissions can be reduced with respect to

${ }^{3}$ The Conference of the Parties serving as the meeting of the Parties to the Kyoto Protocol on its first session (COP/MOP 1) took decisions on a process for considering new binding commitments after the end of the Protocol's first commitment period, for post-2012, for the Kyoto countries. The Conference of the Parties on its eleventh session (COP 11) launched a process on the future of climate change control under the UNFCCC, opening a nonbinding "Dialogue on long-term cooperative action to address climate change by enhancing implementation of the Convention".

${ }^{4}$ Indeed, an increase in recent research efforts emphasises the need to go beyond traditional $\mathrm{CO}$ concentration stabilisation exercises (see e.g., Sarofim et al., 2004; Kemfert et al., 2006; Richels et al., 2004; Tol, 2006).

5 See, for example, the Johannesburg Declaration on Sustainable Development that was adopted at the World Summit on Sustainable Development held in Johannesburg, South Africa, from 2 to 4 September 2002. This statement reaffirms the world's commitment to sustainable development. 
some baseline scenario and/or how a specific GHG target can be achieved in order to stabilise atmospheric concentrations (the so-called QUELRO approach ${ }^{6}$ ). They contain new emission profiles as well as costs and benefits associated with emission reductions. In order to obtain the relevant information that characterises the final outcome, policies are simulated using economic-climate models in order to forecast the potential long-term effects on relevant variables, such as the implied increase in global atmospheric temperature or the effect on GDP growth. By means of such simulations, a comparison of different climate policy scenarios should be possible. Still, given the prevailing scientific uncertainties, an accurate evaluation may need to account for a greater number of indicators and thus comparison of climate policy proposals becomes more difficult.

Therefore, the objective of this paper is not solely to discuss and comment on different policies or policy scenarios, but is primarily to extract useful information in the phase where proposed and simulated climate policy scenarios are compared. This phase is usually crucial for the path the negotiations follow. For this purpose, we apply the Data Envelopment Analysis (DEA), a methodology which is technically closely related to Multi-Criteria Analysis, in that it allows us to deal with situations where multiple inputs and outputs occur. In particular, we are interested in incorporating the economic, environmental and social dimensions of the positive and negative impacts of each policy scenario, in order to bridge the gap between the simulation phase, in which long-run effects of policies are mimicked, and the valuation phase, in which usually a coherent cost benefit analysis framework is adopted. Indeed, these phases should culminate by including the information obtained back into the policy -designing process.

DEA uses data observations to directly evaluate the relative performance of a set of policy scenarios, in a multi input -multi output context. At first, DEA was developed to evaluate the relative efficiency of firms by transforming multiple inputs into multiple outputs, making minimal prior assumptions about the shape of the production possibility set, but inferring information from the data set. While the conventional definition of efficiency can be traced back to Farrell (1957), the first publication that made the DEA methodology popular and introduced it into the operation research world was Charnes

${ }^{6}$ QUELRO stands for "Quantified Emission Limitation and Reduction Objective" and its terminology has been introduced by the Kyoto Protocol. 
et al. (1978). Subsequently, DEA has been applied to evaluate the relative performance of medical services, as in Nyman and Bricke (1989), or of educational institutions, as in Charnes et al. (1981). It has also been applied in the private sector, as in the valuation of banks, in Charnes et al. (1990) ${ }^{7}$. Applications to environmental and resource management problems are less frequent. In general, environmental and social impacts can be modelled as undesirable outputs or as conventional inputs. The absence of market prices for these undesirable outputs, which is a generally recognised valuation problem, can be overcome by employing DEA ${ }^{8}$. However, to the authors' knowledge, DEA has not yet been applied in the comparative assessment of (climate) policies.

The present paper applies this technique in order to evaluate ten climate policy scenarios and finds how scenarios that would not pass the economic efficiency test might represent a good performance in terms of sustainability notwithstanding their relatively high costs.

The rest of the paper is organised as follows. Section 2 introduces the methodological framework describing, on one hand, the model adopted to simulate longrun effects of the different policies, the WITCH model, and on the other hand, the Data Envelopment Analysis (DEA) methodology, which is then applied to compare the various policy scenarios. In particular, the choice of cost and benefit indicators for each policy is introduced and discussed. Section 3 provides a detailed overview of the policy scenarios that are the subject of this analysis. Both the features of the policy proposals and their underlying motivations are tackled. Finally, Section 4 discusses the results and provides conclusions as well as indications for further future research.

\footnotetext{
${ }^{7}$ A thorough review of the theory and applications related to DEA can be found in Coelli et al. (1989), while an extensive bibliography is reported inthe survey articles by Seiford (1996).

${ }^{8}$ Some studies have applied DEA in measuring ecological efficiency (e.g. Dyckhoff and Allen, 2001); some others in measuring the environmental impact of different production technologies, as for example De Koeijer et al. (2002), where the impact of different production techniques in the farm industry are compared. Bosetti and Locatelli (2005) consider the economic and environmental dimensions of management performances of National Parks, while Hernandez-Sancho et al. (2000) consider the issue of efficiency in environmental regulation. An interesting overview of the role of DEA in environmental valuation can be found in Kortelainen and Kuosmanen (2007), while a survey of indicators of firms' environmental behaviour can be found in Tyteca (1996).
} 


\section{Methodology}

The proposed analysis $\$$ based on a composed methodology, as sketched in Figure 1, which couples a traditional simulation analysis - performed in our case by means of a hybrid optimal growth economic-climate model, the WITCH model - with a relative efficiency valuation technique, namely the DEA.

This methodology allows comparing a set of policy scenarios; some of those stemming from political feasibility considerations, some others from scientific concerns regarding global warming and some others from a combination of the two. Motivations behind the design of each policy scenario both in its short and long term features are discussed in greater detail in the subsequent section. We shall now focus on the methodological issues.

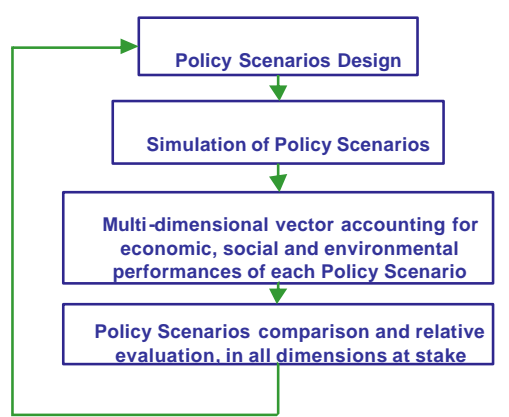

Figure 1. A sketch of the hybrid methodology

Let us start by briefly discussing the simulation phase. Scenarios should be simulated using one, or better, several simulation and/or optimization models (as in the case of the SRES scenarios ${ }^{9}$ or several other comparison exercises). For the sake of simplicity, in the present paper architectures are simulated using one single model.

\footnotetext{
${ }^{9}$ Based on an extensive assessment of the literature, a range of modeling approaches, and a close interaction with many groups and individuals, the IPCC has developed a set of long-term emission scenarios which are described in the IPCC Special Reports on Emission Scenarios (SRES). These scenarios cover a wide array of driving forces of future emissions, and encompass different future developments that might influence GHG sources and sinks. The most recent set of scenarios was published in 2000, more information is available at http://www.grida.no/climate/ipcc/emission/
} 
WITCH - World Induced Technical Change Hybrid model - is a regional integrated assessment model structured to provide normative information on the optimal responses of world economies to climate damages and to model the channels of transmission of climate policy to the economic system. It is an hybrid model because it combines features of both top-down and bottom-up modelling: the top-down component consists of an inter-temporal optimal growth model in which the energy input of the aggregate production function has been expanded to give a bottom-up like description of the energy sector. World countries are grouped in 12 regions that strategically interact following a game theoretic structure. A climate module and a damage function provide the feedback on the economy of carbon dioxide emissions into the atmosphere. WITCH top-down framework guarantees a coherent, fully intertemporal allocation of investments that have an impact on the level of mitigation - R\&D effort, investment in energy technologies, fossil fuel expenditures. The regional specification of the model and the presence of strategic interaction among regions - through $\mathrm{CO}_{2}$, exhaustible natural resources, and technological spillovers - allow us to account for the incentives to free-ride. Applying an open-loop Nash game, the investment strategies are optimized by taking into account both economic and environmental externalities. In WITCH the energy sect or has been detailed and allows a reasonable characterization of future energy and technological scenarios and an assessment of their compatibility with the goal of stabilizing greenhouse gases concentrations. Also, by endogenously modelling fuel (oil, coal, natural gas, uranium) prices, as well as the cost of storing the $\mathrm{CO}_{2}$ captured, the model can be used to evaluate the implication of mitigation policies on the energy system in all its components. For a throughout description of the model see Bosetti et al. (2006) and Bosettiet al. (2007).

The model simulates each climate policy scenario, thus providing the ingredients for the subsequent comparison phase. A set of indicators accounting for performances in different dimensions is stored for each simulated scenario; particularly relevant is information concerning its economic, social and environmental performances. In principle, in the case when more than one model has been simultaneously used to simulate each policy scenario, multiple values for each indicator should be stored. The comparison phase is obviously extremely sensitive to the choice of indicators used to represent 
different dimensions of sustainability. The choice may depend on what features one would like to emphasise and/or on what features the model accounts for. Models with aregional detail can provide information on the distribution of the burden across the world of positive and negative impacts. Obviously, this cannot be accounted for in world aggregate models, even though the issue of un even distribution is recognised as one of the most problematic features of the climate change issue. Besides, models with a detailed description of different climate change impacts ${ }^{10}$, can provide a wider set of environmental indicators.

Given a set of indicators - the reader is referred later in the paper for a detailed discussion on indicators used in the present analysis and reasons behind their choice - it is not always univocally possible to assess which are the most promising policies, unless one weakly dominates all the others (i.e. a policy is superior in at least one dimension without being inferior in any of the remaining dimensions). This depends on the fact that no straightforward way s of aggregating different impacts exist. Therefore, even though a set of modelling groups is asked to report to an international organization, say the IPCC, on long term implications of different policy scenarios, results might not be straightforward to be read. The DEA approach overcomes the problem of incomparability in that it endogenously infers a set of weights by enveloping the data. Note that DEA is extended from its traditional application, namely the evaluation of production firms' performances, to evaluating the performance of policies. Thus, terms such as imuts and outputs, traditionally adopted in the DEA framework, have to be understood here in a broader sense as indicators of costs (or whatever indicators for which lower values are preferred) and indicators of benefits.

There exist two possible interpretations of performing Data Envelopment Analysis to compare the sustainability of different policy scenarios. According to the first interpretation, for each scenario the net economic impact, expressed in monetary value, is aggregated through weights to the social and environmental impacts, expressed in their own unitary measures. DEA is applied in order to obtain the weights by "letting the data speak". The second interpretation, nearer to traditional DEA applications, implies computing a relative efficiency measure for each policy, where efficiency is measured as the ratio

\footnotetext{
${ }^{10}$ For example, information on the impact on sea-level rise could be included in the analysis. See Roson et al. (2004).
} 
of the weighted sum of outputs (i.e., indicators whose value is better to maximize) to the weighted sum of inputs (i.e., indicators measuring features that are optimal to minimize). In particular, a policy is 100 percent efficient if and only if:

- none of its output can be increased without either increasing one or more of its inputs, or decreasing some of its other outputs;

- none of its inputs can be decreased without either decreasing some of its outputs or increasing some of its other inputs.

Let us now consider for the sake of clarity a simple numerical example of five policy scenarios, denoted in Figure 2 as A, B, C, D and E, each using different combinations of two indicators to be minimized (input), say some inequity indicator and the level of temperature increase, and one output to be maximized, say some measure of global welfare. In order to facilitate comparisons, input levels are expressed per unit of output. Data are plotted in Figıre 2. A kinked frontier is drawn from A to C to D, representing the set of relatively efficient scenarios, and the frontier envelopes all these data points and approximates a smooth efficiency frontier. Scenarios on the efficient frontier are assumed to be efficient relatively to scenarios B and D which are considered to be less efficient. It is always possible then to find some projection on the efficient frontier of inefficient scenarios (see B' in Figure 2), thus providing information on potential levers that would improve the designing of inefficient policy scenarios. 


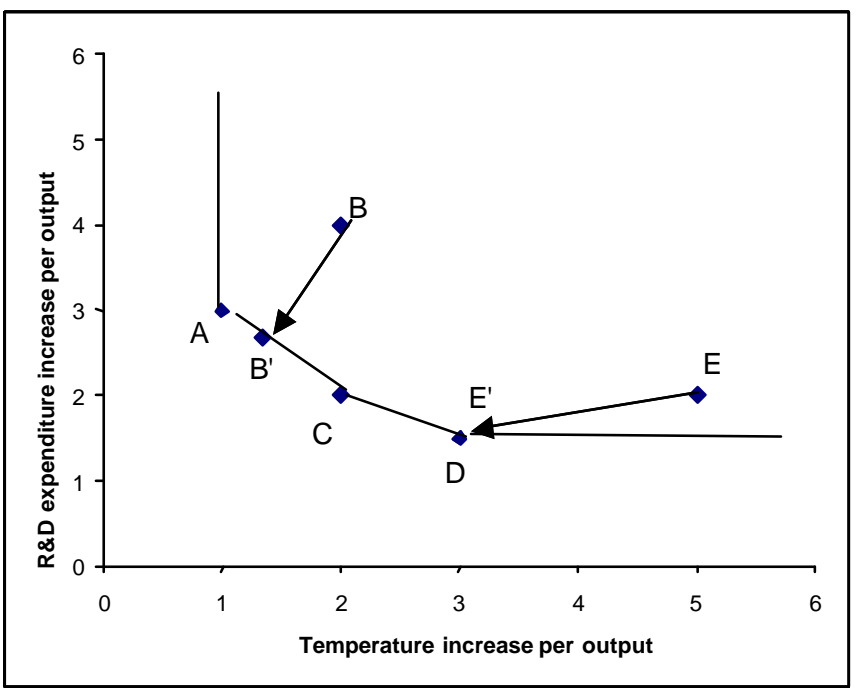

Figure 2. The relative efficient frontier

As discussed above, a choice has to be made on what indicators to use in order to evaluate each simulated scenario. An indicator accounting for the global intertemporal economic performance of the scenario needs to be defined. In our application we propose a global welfare indicator for each policy scenario defined as the present value of GDP summed over all world regions. In addition, each scenario should be characterised by a social impact indicator and an environmental impact indicator. In our application, to account for the social perspective, we value each scenario for its impact on the distribution of income among different regions of the world, measured using an equity index. The choice of a certain measure of inequality is a debated one, because it always involves the introduction of specific value judgements; see Atkinson (1970) for a detailed discussion of this topic. In our work we adopt the Gini indicator to account for the equity level reached by the end of the century in each different scenario.

Finally, in order to account for the environmental impact of each policy once more one faces the choice among several alternative measures; e.g carbon or GHGs emissions, carbon or GHGs atmospheric concentrations, increase in radiative forcing or in temperature or total damage. For the sake of simplicity in the present study we employ the increase in temperature above pre-industrial levels in degree Celcius. 


\section{DEA-based Cost Benefit Analysis.}

Following the first interpretation of the DEA, we can define total benefit, $T B_{m}$ of policy $m$ as the difference between the global welfare index, $N B_{m}$, and the environmental, $Z_{e, m}$ and social, $Z_{s, m}$ impacts, more formally:

$$
T B_{m}=N B_{m}-p_{e} Z_{e, m}-p_{s} Z_{s, m}
$$

where $p_{e}$ and $p_{s}$ are the weights associated to the environmental and social indices. As extensively discussed in Kuosmanen and Kortelainen (2007), we can consider the problem from a gametheoretic perspective and suppose that supporters of a particular policy can adopt a strategic opportunistic behaviour and propose ${ }^{11}$ the weight vector, $\mathbf{p}$, which maximises the advantages of their supported policy over that of the others. In practice, this consists of solving, for each policy $m$, the problem of choosing a non negative weight vector that maximises that policy's Comparative Advantage $\left(C A_{m}\right)$, defined as the (minimum) multi-dimensional distance to all other policy's total benefits. More formally, it consists of solving, for each of the analysed policies, $m \in M$, the following linear programming problem:

$$
\begin{aligned}
& \max _{p_{e}, p_{s}} C A_{m} \\
& \text { s.t. } \\
& C A_{m} \leq\left[N B_{m}-p_{e} Z_{e, m}-p_{s} Z_{s, m}\right]-\left[N B_{1}-p_{e} Z_{e, 1}-p_{s} Z_{s, 1}\right] \\
& C A_{m} \leq\left[N B_{m}-p_{e} Z_{e, m}-p_{s} Z_{s, m}\right]-\left[N B_{2}-p_{e} Z_{e, 2}-p_{s} Z_{s, 2}\right] \\
& \mathrm{M} \\
& C A_{m} \leq\left[N B_{m}-p_{e} Z_{e, m}-p_{s} Z_{s, m}\right]-\left[N B_{m-1}-p_{e} Z_{e, m-1}-p_{s} Z_{s, m-1}\right] \\
& C A_{m} \leq\left[N B_{m}-p_{e} Z_{e, m}-p_{s} Z_{s, m}\right]-\left[N B_{m+1}-p_{e} Z_{e, m+1}-p_{s} Z_{s, m+1}\right] \\
& \mathrm{M} \\
& C A_{m} \leq\left[N B_{m}-p_{e} Z_{e, m}-p_{s} Z_{s, m}\right]-\left[N B_{M}-p_{e} Z_{e, M}-p_{s} Z_{s, M}\right] \\
& N B_{m}-p_{e} Z_{e, m}-p_{s} Z_{s, m} \geq 0 \\
& p_{e}, p_{s} \geq 0
\end{aligned}
$$

When the $m$-th policy turns out not to have any comparative advantage over the others, $C A_{m}<0$, even allowing for the choice of the most convenient weight vector, then the policy should be rejected, according to the chosen set of indicators. Contrariwise, for policies showing a non-negative comparative

\footnotetext{
${ }^{11}$ As an example, on the basis of results of valuation studies, opportunely and opportunistically manipulated.
} 
advantage over other policies $C A_{m} \geq 0$, a sensitivity analysis on weights should be performed in order to obtain more information. Indeed, letting one or both weights be set to zero, in order to magnify the deriving comparative advantage of the policy, implies that one or both dimensions, the environmental and social one, have been dropped out from the valuation procedure. The process can be enhanced by interfacing the discussion on the domain of weights to the political debate, thus enabling policy makers to gain a better understanding of how to interpret analysis results.

\section{DEA relative efficiency computation.}

The second approach involves the computation of relative efficiency scores. A maximum score of unity (or 100\%) is considered as the benchmark. Indicators are now reinterpreted in terms of inputs (cost or in general indicators which is preferable to minimize) and outputs (benefits or in general indicators which is preferable to maximize). Both inputs and outputs may belong to the economic, environmental or social dimension. In our application, we consider on the input side the social and environmental indicator. Both the Gini indicator and the global atmospheric temperature should be minimized Instead, on the output side, we consider global discounted GDP, which should be maximized.

The efficiency score of each policy scenario is expressed as the ratio of the weighted sum of outputs over the weighted sum of inputs. For each policy, a set of weights is chosen such that it maximises its efficiency. More formally, given the set of $M$ policies, each with $J$ outputs (benefits) given a set of $I$ inputs (costs), let us denote by $y_{j m}$ and $x_{i m}$ the vectors representing the quantities of outputs and inputs relative to the $m$-th DMU, respectively. The efficiency of the $m$-th policy can thus be calculated as:

$$
e_{m}=\frac{\sum_{j=1}^{J} u_{j} y_{j m}}{\sum_{i=1}^{I} v_{i} x_{i m}}, \quad\left[\begin{array}{l}
j=1, . ., J \\
i=1, . ., I
\end{array}\right]
$$

where $u_{j}$ and $v_{i}$ are two vectors of weights used in the measurement of policy $m$ 's relative importance of inputs and outputs calculated through the maximisation problem, which is stated below for policy $m$ : 


$$
\begin{aligned}
& \max _{u_{j}, v_{i}} e_{m} \\
& \text { s.t. } \\
& \frac{\sum_{j=1}^{J} u_{j} y_{j n}}{\sum_{i=1}^{I} v_{i} x_{i n}} \leq 1 \quad \forall n=1, ., m, ., M \\
& 0 \leq u_{j} \leq 1 \\
& 0 \leq v_{i} \leq 1
\end{aligned}
$$

Weights must be constrained to take strictly positive values (greater than or equal to a very small quantity epsilon) so that no inputs or outputs are ignored in the process of determining the efficiency of each policy.

If the solution to the maximisation problem gives a value of efficiency equal to 100 , the corresponding scenario is considered to be efficient or non-dominated; if instead the efficiency value is inferior to 100 , then the corresponding scenario is said to be dominated, and therefore does not lie on the efficiency frontier, which is defined by the envelopment of efficient scenarios. Furthermore, information concerning potential improvements of inefficient policies can be obtained.

\section{Ten Climate Policy Scenarios}

Based on the above methodological framework, a number of policy scenarios can be evaluated. This section will introduce the policy scenarios that have been chosen for our analysis, and describe the relevant policy framework as well as the reasons behind their choice. In total, we have designed ten scenarios on the basis of indications from policy processes and the scientific community. These indications represent the "criteria" for why policy scenarios have been chosen. The resulting scenarios are particularly relevant for potential considerations of future climate policy, and cover a series of potential future perspectives. The number of scenarios is arbitrary; given the overall objective of appropriately testing a new evaluation tool, a wide range of both more and less likely options are essential. In addition to these policy scenarios, we provide as a first scenario a business-as-usual projection in order to have a credible benchmark for our evaluation. The BAU scenario is characterised by a continuation of the current trends in the main economic and environmental parameters. 
The remaining ten policy scenarios possess some common features. In particular, all scenarios assume that the absolute emission reductions defined in the Kyoto Protocol will be achieved by the Annex $B_{-U s}$ countries ${ }^{12}$ by 2010 (first commitment period). Indeed, it was Russia's ratification of the Kyoto Protocol on November 4, 2004 that opened the way for the Protocol's coming into force on February 16, 2005, making thus the emissions targets taken on for the 2008-2012 period by more than 30 developed countries (including the EU, Russia, Japan, Canada, New Zealand, Norway and Switzerland) legally binding ${ }^{13}$ According to its domestic policy target, he US is assumed to achieve its $-18 \%$ emission intensity target in order to slow the grow th of GHG emissions per unit of economic activity over the next 10 years ${ }^{14}$. Developing countries have no target in the first commitment period.

Then, different assumptions characterise the different scenarios from 2020 onwards. ${ }^{15}$.

The second scenario assumes a continuation of the current situation. After the US announced its defection from the Kyoto Protocol in March 2001, the remaining Kyoto countries - in particular the EU and Japan - put great effort into the continuation of the Kyoto process, in particular by convincing Russia to participate in the Protocol. After meeting the Kyoto objectives at the end of the first commitment period, this scenario assumes that the Annex $\mathrm{B}_{-\mathrm{US}}$ countries decide to maintain their initial Kyoto targets and thus the corresponding emission level until the year 2100, whereas the US remains out of the Kyoto Protocol and implements no effective climate policy. This scenario thus represents the situation in which the Annex $\mathrm{B}_{- \text {us }}$ countries behave according to the "Kyoto forever" hypdhesis, whereas the US and the developing countries have no binding emission constraints. All countries adopt cost-effective environmental policies, and in particular, emissions trading takes place among the Annex $\mathrm{B}_{-\mathrm{US}}$ countries.

\footnotetext{
${ }^{12}$ We denote by Annex $\mathrm{B}_{- \text {US }}$ the countries listed in the Annex B of the Kyoto Protocol without the participation of the United States.

13 The Kyoto Protocol imposes absolute reduction targets, i.e. a reduction of absolute GHG emissions by a specified percentage.

${ }^{14}$ In order to replicate the US strategy as precisely as possible, our model computes the $-18 \%$ intensity reduction by 2010 compared to the year 2000. Climate policy in terms of emission intensity targets is typically expressed as percentage reductions from some base year level. In the US context, greenhouse gas intensity is given by the ratio of greenhouse gas emissions to economic output.

${ }^{15}$ Note that scenarios 2 to 7 , chosen to cover both optimistic and pessimistic predictions on future abatement targets, have already been discussed in greater detail in Buchner and Carraro (2007). In particular, using the integrated climate-economy model FEEM-RICE, the six different scenarios on future emission abatement commitments have been analysed to provide an assessment of their implications for the economy. However, given the different scope of this paper, we will briefly recall their main features in order to enable a comprehensive background to our analysis.
} 
In the third scenario we assume that, given international and domestic political pressures, the US decides to join the group of countries committed to the Kyoto Protocol in the second commitment period and afterwards. Continuity with Kyoto could be attractive for the countries that are already engaged in the Kyoto Protocol, i.e. the Annex $B_{- \text {us, }}$ since these countries have already made a substantial investment in the Kyoto process (Bodansky, 2003). Developing countries, as in "Kyoto forever", are assumed not to adopt any emission target until 2050. Consequently, emissions in Annex B countries will be stabilised at about $-5 \%$ by 2100 w.r.t their 1990 value, whereas emissions in developing countries will keep growing.

Common assumptions characterise the second commitment period (2010-2020) of scenarios 47. International and domestic pressures for climate change control are expected to induce countries to further strengthen their efforts in international climate policy (Cf. IPCC WG1, 2007). In particular, both the remaining Annex B countries and the US are assumed to agree by 2020 to reduce emissions by an additional $20 \%$ compared to the level of emissions in 1990 . The $-20 \%$ objective for developed countries appears a possible scenario given recent policy signals. On one hand, the European Commission presented its Integrated Energy and Climate Package on 10 January 2007 with the objective of establishing a new energy policy for Europe to combat climate change and boost at the same time the EU's energy security and competitiveness ${ }^{16}$. Amongst others, the Commissions put forward plans on ambitious targets on GHG emissions: a reduction target of $-30 \%$ in GHG emissions from developed countries by 2020 is proposed if an international agreement will be reached. In alternative, a unilateral EU emissions reduction target of at least $20 \%$ by 2020 is proposed $^{17}$. These targets have subsequently been supported by the EU environmental ministries of the EU member states. ${ }^{18}$ On the other hand, a $10 \%$ target for developed countries was indicated as the most likely one for the second commitment period by

16 The communication from the Commission on "An energy policy for Europe" can be downloaded at http:/leurlex.europa.eu/LexUriServ/site/en/com/2007/com2007_0001en01.pdf

${ }^{17}$ The Communication by the Commission on "Limiting Global Climate Change to 2 degrees Celsius - The way ahead for 2020 and beyond" can be downloaded at http://ec.europa.eu/environment/climat/pdf/future_action/com_2007_2_en.pdf

${ }^{18}$ At the Council meeting in February 2007, the environment ministers agreed on the emission reduction targets proposed by the European Commission in January 2007, showing the willingness to commit to a reduction of $30 \%$ of GHG emissions by 2020 compared to 1990, "provided that other developed countries commit themselves to comparable emission reductions and economically more advanced developing countries adequately contribute according to their responsibilities and respective capabilities" and suggesting that the EU should reduce greenhouse gas emissions by 20 per cent below 1990 levels by 2020 . This last conclusion will also be addressed at the Spring European Council (8-9 March 2007). Further details are provided under http://europa.eu/rapid/pressReleasesAction.do?reference=PRES/07/25\&format=HTML\&aged=0\&language=EN\&guiLanguage $=$

en 
a panel of 78 experts interviewed by Böhringer and Löschel (2003). Taking into account all these signals, a 20\%-target for industrialised countries appears a likely compromise. In order to account for the need for developing countries to continue their economic and social development, they are still exempt from complying with emission reduction targets. This assumption is also in line with recent research studies which conclude that it is unlikely developing countries will be included in international climate change control agreements before $2020^{19}$ as well as with indications from the policy process (for instance arising from the "Dialogue on longterm cooperative action to address climate change by enhancing implementation of the Convention" that has been launched under the UNFCCC).

In the fourth scenario therefore, the Annex $\mathrm{B}_{-\mathrm{US}}$ countries achieve the Kyoto target in the first commitment period and the $-20 \%$ target (w.r.t. 1990 emissions) in the second one. The US adopts its $18 \%$ intensity target in the first commitment period and the $-10 \%$ absolute target (w.r.t. 1990 emissions) in the second one. Developing countries do not commit to any emission reductions. After 2020, we assume that cooperation on climate change control collapses and emissions return to their business-asusual (BAU) paths.

The fifth scenario is based on the idea that Kyoto targets are largely sub-optimal - i.e. the incentives to reduce carbon emissions should be great enough to reach more ambitious targets - and that countries are only likely to adopt targets closer to the optimal ones in the medium term. The two initial commitment periods stay the same as in scenario 4. Then, Annex B countries (including the US) and developing countries adopt what we call "enhanced permanent cooperation emission targets", computed as follows. All countries cooperatively maximise their joint welfare with respect to their policy variables, including GHG emissions. This yields the optimal path of GHG emissions in all world regions, as it represents the cooperative outcome to all nations. Then, on the basis of the precautionary principle and given the relatively low emissions reduction in their optimal strategy, all countries pledge to reduce their emissions by an additional 10\% below the optimal emission trajectories from 2020 onward.

\footnotetext{
${ }^{19}$ For example, expert judgements presented in Böhringer and Löschel (2003) reveal that in the second commitment period up to 2020 “...in $75 \%$ of the policy-relevant scenarios, developing countries do not commit themselves to binding targets." (p. 9)
} 
The sixth scenario starts from the same premise as the previous one, namely that serious emission reductions are essential. Therefore, developed countries are assumed to follow the suggestion by the European Commission, e.g. to reduce their GHG emissions by $30 \%$ compared to their 1990 emissions by 2020. Starting from 2020, the so-called Kyoto countries - Japan, European Union and Russia - are supposed, by 2050 , to have achieved a total reduction in GHG emissions of $-70 \%$ with respect to their 1990 emissions. This target is based on the recommendations of several politicians regarding the dangers of climate change. For example, the English Prime Minister Tony Blair has proposed to aim at a $60 \%$ cut in carbon emissions by 2050 , thus implementing an emission reduction target of $-10 \%$ for each decade, and he has advocated this target for all industrialised countries. ${ }^{20}$ Also the French President Chirac echoed Blair's proposal and insisted on a strong commitment to reduce GHG emissions. ${ }^{21}$ In a recent announcement, the European Council re-affirmed this intention, although the ambitious goal has not yet been supported by an agreed statement ${ }^{22}$. The other countries - the US and the developing regions reduce their emissions between 2020 and 2050 by $-15 \%$ for each decade. These targets thus imply strong emission reductions in both the US and the developing countries. From 2050 onwards, when countries have achieved the ambitious emission levels, all nations are committed to maintaining these emission levels.

Scenarios 7, 8 and 9 are based on the common view that a stabilisation level of $550 \mathrm{ppmv}^{23}$ in 2100 represents a reasonable goal, also adopted in the emission mitigation scenarios examined by the Third IPCC report (IPCC, 2001) ${ }^{24}$. In particular, the analysis of Working Group III in the TAR suggests

${ }^{20}$ The reduction goal is based on the outcomes of a report by The Royal Commission on Environmental Pollution (2000) which found that a $60 \%$ reduction by 2050 was essential if the overall goal of stabilising GHG emissions at 550 ppmv was to be achieved already by 2050 .

${ }^{21} \mathrm{Mr}$. Chirac emphasised the need for action to combat climate change again at a recent conference in January 2007. See for instance http://www.ft.com/cms/s/52e2cef2-b4bd-11db-b707-0000779e2340.html

22 The 25 ministers agreed on March 232005 that developed nations should pursue cuts of heat-trapping gases of 15-30 percent by 2020 and 60-80 percent by 2050 compared with levels set in the Kyoto Protocol, which uses 1990 as a bas e in most cases. But the longer-term 2050 goal has been omitted from an agreed statement. At the Council meeting in February 2007, the environment ministers agreed on the emission reduction targets proposed by the European Commission in January 2007, but made no progress on the 2050-target.

${ }^{23}$ Parts per million by volume is a measure of concentration of gases in the atmosphere.

${ }^{24}$ The target of not exceeding the 550 ppmv concentration level is also supported by the EU. The first significant EU proposal for a climate target for the post-2000 period, presented at the EU Council of Ministers in 1996, suggested stabilising the atmospheric concentrations of $\mathrm{CO}_{2}$ at a level around twice the pre-industrial level of about $280 \mathrm{ppmv}$, corresponding thus to the concentration target of 550 ppmv. 
that achieving the aggregate Kyoto commitments in the first commitment period can be consistent with trajectories that achieve stabilisation at 550 ppmv by the end of the century (WGIII TAR, Section 2.5.2). This concentration level also coincides with a doubling of $\mathrm{CO}_{2}$ atmospheric concentrations compared to pre-industrial levels, implying a global warming of up to $3^{\circ} \mathrm{C}$ with a change in the mean surface temperature in the range of $1.6^{\circ} \mathrm{C}-2.9^{\circ} \mathrm{C}$ by $2100^{25}$. This long-term goal is imposed from the second commitment period onwards, from 2020 to 2100 , and is to be achieved through various means of burdensharing.

In the seventh scenario we assume that all countries agree to make substantial efforts to control GHG emissions and to stabilise global GHG emissions at $550 \mathrm{ppmv}$ in the year 2100. As indicated, this concentration goal is often used as a baseline hypothesis for models examining climate sensitivity. We assume linear convergence to $550 \mathrm{ppmv}$ in 2100 , starting in 2020 . The two initial commitment periods are designed as in Scenario 6. From 2020 onwards, targets are calculated to achieve the 550 ppmv stabilisation goal. This global target is allocated among the different world regions according to the "sovereignty" equity rule, as suggested by 78 experts Böhringer and Löschel (2003). This rule requires that the emission entitlements are shared in proportion to emissions, current emissions constitute thus a type of status quo at the moment. Therefore, the emission targets for 2030, 2040 and 2050 for all world regions, including developing countries, are based on both the 550 ppmv stabilisation goal and the sovereignty rule.

The eighth scenario is based on the so-called Brazilian Proposal, made for the first time by Brazil during the negotiations on the Kyoto Protocol. The idea is to allocate the emissions reductions of the industrialised, Annex I Parties in relation to the relative effect of a country's historical emissions on global temperature increase (UNFCCC, 1997). We use this approach as suggested by RIVM (Cf. del Elzen et al., 2003), i.e. the Brazilian Proposal is applied on a global level from 2010, combined with a threshold for participation for the non-Annex I regions. In particular, the stabilisation of atmospheric concentrations at $550 \mathrm{ppm}$ in 2100 is achieved through burden sharing based on the contribution to

\footnotetext{
${ }^{25}$ In this range, although the strongest effects of climate change can be prevented, potentially serious damage attributable to climatic changes could still occur.
} 
temperature increase, combined with an income threshold for participation of the non-Annex I regions. This participation threshold is chosen as a percentage of the 1990 PPP Annex I per capita income.

The ninth scenario applies an allocation concept proposed by the Global Commons Institute, the so-called Contraction \& Convergence approach (Meyer, 2000), to stabilise atmospheric concentrations at $550 \mathrm{ppm}$ in 2100. This burden-sharing rule is also known as the Per Capita Convergence (PCC) approach and defines emission permits on the basis of a convergence of per capita emissions under a contracting global GHG emission profile. In such a convergence regime, all countries participate in the climate regime with emission allowances converging to equal per capita levels over time.

The last two scenarios take into account the immense dangers embedded in a potential climate change. They are thus derived from the scientific perspective of climatologists who claim that strong emission reductions are required in order to halt the threat of global warming.

In particular, the tenth scenario is based on the aim to stabilise $\mathrm{CO}_{2}$ concentrations at $450 \mathrm{ppmv}$ by 2100 . This emission reduction target is in general considered to be quite stringent, because it limits global mean warming to less than $3^{\circ} \mathrm{C}$ (see e.g. the analysis by the Working Group III in IPCC, 2001), making thus the achievement of the often-cited $2^{\circ} \mathrm{C}$ target possible. Indeed, such a stabilisation is supposed to significantly reduce or even avoid many of the impacts listed for $3^{\circ} \mathrm{C}$ warming or more, enabling thus much higher benefits than a stabilisation at lower levels. ${ }^{26} \mathrm{We}$ assume linear convergence to 450 ppmv in 2100, starting in 2020, again according to the lines of Contraction \& Convergence.

Table 1 provides a comprehensive summary of the main features of the 10 scenarios.

\section{Discussion of results and conclusions}

For the purpose of this paper, we have simulated a set of ten policy scenarios, computed the three indicators for each of them (cumulated discounted GDP over the century, temperature increase by 2100 , and Gini equity indicator by 2100), and finally applied DEA in its two possible interpretations. As mentioned above, the idea is to have each scenario evaluated as its supporters would do, thus selecting weights to aggregate indicators in the most favourable way for that scenario. While the evaluation of a

${ }^{26}$ Note, however, that there would still be risks for impacts associated with mean warming of less than $3^{\circ} \mathrm{C}$. 
policy is typically made on the basis of its short/long term economic or environmental performance solely, this approach allows accounting for more dimensions contemporaneously. Results derived from the two DEA interpretations are very similar, but we present both of them in order to emphasize the potentials of each of the two approaches.

Let us first consider the ranking of scenarios shown in Table 2. There appear to be three winners, namely three policies at least weakly dominating all the others: "Kyoto-US only in 1st commitment period”, "550ppmv through the RIVM's Brazilian Proposal”, “-70\% emission target”. If we grouped the scenarios in three categories, low (Scenarios 1 to 5), medium (Scenarios 7 to 9) and high environmental effectiveness (Scenarios 6 and 10), these three policies represent the best performance within each of the three categories, in terms of costs and of equity/wealth redistribution.

Let us analyze these three policies in detail. The attractiveness of the first one, "Kyoto-US only in 1st commitment period", comes at no surprise. In addition to a modest environmental target, it also entails very low costs (GDP remains therefore as high as in the business as usual throughout the century). This depends on the fact that although the policy entails some costs, most of them are outweighed by the benefits derived through the internalization of the climate change externality, in primis, and other externalities as well, as for example the ones depending on the excessive use of natural resources and those stemming from the underinvestment in R\&D. Of course, in terms of avoided temperature increase this policy is not extremely effective (by the end of the century temperature has increased of $2.5^{\circ} \mathrm{C}$ versus the $2.75^{\circ} \mathrm{C}$ increase that would occur in the baseline). However, some redistribution of wealth takes place because effort is undertaken by Annex 1 countries only while other countries will mainly benefit from the policy. This entails a better performance in terms of equity with respect to all other scenarios within the low environmental effectiveness ones.

The second scenario obtaining the highest score, the "550ppmv through the RIVM's Brazilian Proposal", represent a slight improvement in terms of stringency of the climate target (even though a stabilization of atmospheric $\mathrm{CO}_{2}$ at $550 \mathrm{ppmv}$ implies with a high likelihood a temperature above the $2^{\circ} \mathrm{C}$ target). Although costs are higher than for the low environmental effectiveness set of scenarios, they are contained in the sense of not being excessive. On the other side, the redistribution potential entailed by 
the proposed allocation scheme makes this scenario the most equitable among the medium environmental effectiveness ones. These reasons together, i.e. the limited costs, the medium environmental effectiveness and the high social performance, render this scenario one of the winners. This suggests that even an involvement of developing countries in the short term might be possible, if the specific climate policy package is well designed.

Finally, the third winner is the "-70\% emission target" scenario that entails a major difference from the other two because of its environmental stringency. There is an effective impact on temperature increase $\left(1.6^{\circ} \mathrm{C}\right.$ by 2100$)$ which makes this scenario the one performing best in environmental terms. Of course, this strong environmental effectiveness comes at a high cost (even higher than in the case of the 450 ppmv stabilization). At the same time, however, the way the burden of this climate policy is distribut ed among world regions implies a large wealth redistribution, which enables this scenario also to have the best performance in social terms. This result is interesting because it indicates that a stringent climate policy can become an attractive option if all sustainability impacts are taken into account.

The comparative advantage analysis (results are provided in Table 3) enables to investigate results for different lower bounds on weights, which represent different assumption on the relative importance policy-makers attach to the social and environmental dimensions. Setting the lower bound for weights at zero (last column of Table 3) means that one allows for the possibility of completely neglecting both environmental and social impacts of a policy when valuing its comparative advantage. Even in such an extreme case, where most of the policies pass the test of having a positive comparative advantage, the "BAU" and the "Annex B cooperation until 2020" scenarios show a negative comparative advantage. If one restricts the value of weights to be strictly greater than zero (second column from the right in Table 3), this corresponds to a situation in which policy-makers attach at least some importance to both environmental and social impacts of the policy. In such a case, we find two more scenarios characterized by a negative comparative advantage, "Kyoto-us" and the so-called "Enhanced permanent global cooperation", implying that they involve a low environmental and social performance compared to other scenarios that apparently perform slightly better in one or both dimensions. Finally, letting the minimum value of weights increase (second column in Table 3) implies that social and environmental 
impacts assume increasingly relative importance in policy-makers' value judgment. There exists a threshold value for weights at which the only scenario measuring a positive comparative advantage is the “-70\% emission target" scenario, which has already been described as performing best in social and environmental terms. This policy scenario obtains thus a good performance in terms of sustainability notwithstanding its ambitious environmental target and its related significant costs.

The findings of this analysis need to be taken cautiously, because of the arbitrariness of the choice of indicators and of the simulation model. Nonetheless, it represents an attempt to go beyond the comparison done purely on economic terms that is typically undertaken in order to discuss the advantages of alternative long term climate policies.

This methodology can represent an impotant tool for policy makers, through the identification and evaluation of a number of policy strategies that appear to be crucial for the evolution of the future climate debate. As a consequence, the approach adopted in this paper could be extremely beneficial if combined with different types of climate-economy models and different choices of costs and benefits indicators. 


\section{References}

Atkinson, A.B., 1970. On the Measurement of Inequality . Journal of Economic Theory, 2: 244-263.

Barrett, S., 1994. Self-Enforcing International Environmental Agreements. Oxford Economic Papers, 46: 878-894.

Bodansky, D., 2003. Climate Commitments: Assessing the Options. Pew Center on Global Climate Change. In: Aldy et al., Beyond Kyoto. Advancing the International Effort Against Climate Change. Pew Center on Global Climate Change.

Böhringer, C. and Löschel, A., 2003. Climate Policy Beyond Kyoto: Quo Vadis? A Computable General Equilibrium Analysis based on Expert Judgements. ZEW Discussion Paper No. 0309, Mannheim.

Bosetti, V. and Locatelli, G., 2006. A Data Envelopment Analysis Approach To The Assessment Of Natural Parks' Economic Efficiency And Sustainability. The Case Of Italian National Parks. Sustainable Development (2006) DOI: 10.1002/sd.288.

Bosetti, V., Carraro, C., Galeotti, M., Massetti, E. and Tavoni, M., 2006. WITCH: A World Induced Technical Change Hybrid Model. The Energy Journal, Special Issue on Hybrid Modeling of Energy -Environment Policies: Reconciling Bottom-up and Top -down, 13-38.

Bosetti, V., Massetti, E. and Tavoni, M., 2007. FEEM's WITCH Model: Structure, Baseline, Solutions. FEEM Working Paper No 10/2007.

Botteon, M. and Carraro, C., 1997. Burden-Sharing and Coalition Stability in Environmental Negotiations with Asymmetric Countries. In: C. Carraro (Editor), International Environmental Agreements: Strategic Policy Issues, E. Elgar, Cheltenham.

Buonanno, P., Carraro, C., Castelnuovo, E. and Galeotti, M, 2000. Efficiency and Equity of Emission Trading with Endogenous Environmental Technical Change. In: C. Carraro (Editor), Efficiency and Equity of Climate Change Policy. Dordrecht: Kluwer Academic Publishers.

Buchner, B. and Carraro, C., 2004. Emissions Trading Regimes and Incentives to Participation in International Climate Agreements. European Environment, 14 (5): 276-289. 
Buchner, B. and Carraro, C., 2005a. Economic and Environmental Effectiveness of a Technology based Climate Protocol. Climate Policy, 4: 229-248.

Buchner, B. and Carraro, C., 2005b. Modelling Climate Policy. Perspectives on Future Negotiations. Journal of Policy Modeling, 27(6): 711-732.

Buchner, B. and Carraro, C., 2006. US, China and the Economics of Climate Negotiations. International Environmental Agreements: Politics, Law and Economics, 6(1): 63-89.

Buchner, B. and Carraro, C., 2007. Parallel Climate Blocs. Incentives to Cooperation in International Climate Negotiations. In: R. Guesnerie and H. Tulkens (Editors), The Design of Climate Policy. Forthcoming, MIT Press.

Carraro, C., 1998. Beyond Kyoto: A Game Theoretic Perspective. In the Proceedings of the OECD Workshop on "Climate Change and Economic Modelling. Background Analysis for the Kyoto Protocol", Paris, 17-18.9, 1998.

Carraro, C., 1999. The Structure of International Agreements on Climate Change. In: C. Carraro (Editor), International Environmental Agreements on Climate Change, Kluwer Academic Pub.: Dordrecht.

Carraro, C. and Siniscalco, D., 1993. Strategies for the International Protection of the Environment. Journal of Public Economics, 52: 309-328.

Charnes, A., Cooper, W.W., and Rhodes, E., 1978. Measuring Efficiency of Decision Making Units. European Journal of Operational Research, 2 (6): 429-444.

Charnes, A., Cooper, W. W., and Rhodes, E., 1981. Evaluating Program and Managerial Efficiency: An Application of Data Envelopment Analysis to Follow Through. Management Science, 27 (6): 668-696.

Charnes, A., Cooper, W. W., Huang, Z. and Sun, D., 1990. Polyhedral Cone-Ratio DEA Models with an Illustrative Application to Large Commercial Banks. Journal of Econometrics, 46 (1/2): 73-91.

Coelli, T., Prasada Rao, D.S. and Battese, G.E., 1998. An Introduction to Efficiency and Productivity Analysis. Boston: Kluwer Academic Publishers. 
Dyckhoff, H., and Allen, K., 2001. Measuring Ecological Efficiency with Data Envelopment Analysis (DEA). European Journal of Operational Research, 132 (2): 312-325.

De Koeijer, TJ, Wossink, GAA, Struik, PC, Renkema, JA., 2002. Measuring Agricultural Sustainability in Terms of Efficiency: The Case of Dutch Sugar Beet Growers. Journal of Environmental Management, 66 (1): 9 - 17.

Egenhofer, C., W. Hager and Legge, T., 2001. Defining Europe's Near Abroad in Climate Change: A Russian-EU Alliance - Sub-global Bargaining to Further International Environmental Agreements. CEPS Discussion Paper, Brussels.

Farrell, M.J., 1957. The Measurement of Productive Efficiency. Journal of the Statistical Society Series A (General), 120: 253-281.

Green, Michael J.B. and Paine, J., 1997. State of the World's Protected Areas at the End of the Twentieth Century. IUCN.

Hernandez-Sancho, F. Picazo-Tadeo, A and ReigMartinez, E., 2000. Efficiency and Environmental Regulation An Application to Spanish Wooden Goods and Furnishings Industry. Environmental and Resource Economics, 15: 365-378.

Intergovernmental Panel on Climate Change (IPCC), 2001. Climate Change 2001: Mitigation. Cambridge: Cambridge University Press.

Intergovernmental Panel on Climate Change (IPCC), 2007. Climate Change 2007: The Physical Science Basis, Summary for Policymakers. Contribution of Working Group I to the Fourth Assessment Report, http://ipccwg1.ucar.edu/wg1/docs/WG1AR4_SPM_Approved_05Feb.pdf .

Kemfert, C., Truong, T., and Bruckner, T., 2006. Economic Impact Assessment of Climate Change: A Multi-Gas Investigation with WIAGEM -GTAPEL-ICM. The Energy Journal, Special Issue Number 3, 28: 441-460.

Kuosmanen, T. and Kortelainen, M., 2007. Valuing environmental factors in cost-benefit analysis using data envelopment analysis, Ecological Economics, Elsevier, 62(1): 56-65. 
Meyer, A. 2000. Contraction \& Convergence. The Global Solution to Climate Change. Schumacher Briefings, 5. Green Books, Bristol, UK.

Nyman, J.A. and Bricker, D.L., 1989. Profit Incentives and Technical Efficiency in the Production of Nursing Home Care. Review of Economics and Statistics, 71 (4): 586-594.

Richels, R.G, Manne, A.S., and Wigley, T.M.L., 2004. Moving Beyond Concentrations: The Challenge of Limiting Temperature Change. AEI-Brookings Joint Center for Regulatory Studies, Working Paper 04-11.

Roson, R., Bosello, F., Lazzarin, M. and Tol, R., 2004. Economy-Wide Estimates of the Implications of Climate Change: Seal Level Rise. FEEM Working Paper 96.04, Milan.

Sarofim, M.C., Forest, C.M., Reiner, D.M. and Reilly, J.M., 2004. Stabilization and Global Climate Policy. MIT Joint Program on the Science and Policy of Global Change Report No. 110, Cambridge, MA.

Seiford, L.M., 1996. Data Envelopment Analysis: The Evaluation of the State of Art (1978-1995). The Journal of Productivity Analysis, 7: 99-137.

Stewart, R and Wiener, J., 2003. Reconstructing Climate Policy, American Enterprise Institute Press.

The Royal Commission on Environmental Pollution, 2000. Energy - The Changing Climate. 22nd Report.

Tol, R.S.J., 2006. Multi-Gas Emission Reduction for Climate Change Policy: An Application of FUND. The Energy Journal, Special Issue Number 3, 28: 235-250.

Tyteca, D., 1996. On the Measurement of the Environmental Performance of Firms- A Literature Review and a Productive Efficiency Perspective. Journal of Environmental Management, 46: 281-308.

United Nations Framework Convention on Climate Change (UNFCCC), 1997. Implementation of the Berlin Mandate. Additional Proposals from Parties. Addendum FCCC/AGBM/1997/MISC.1/Add.3. Available on http://unfccc.int/resource/docs/1997/agbm/misc01a3.pdf 
Tables

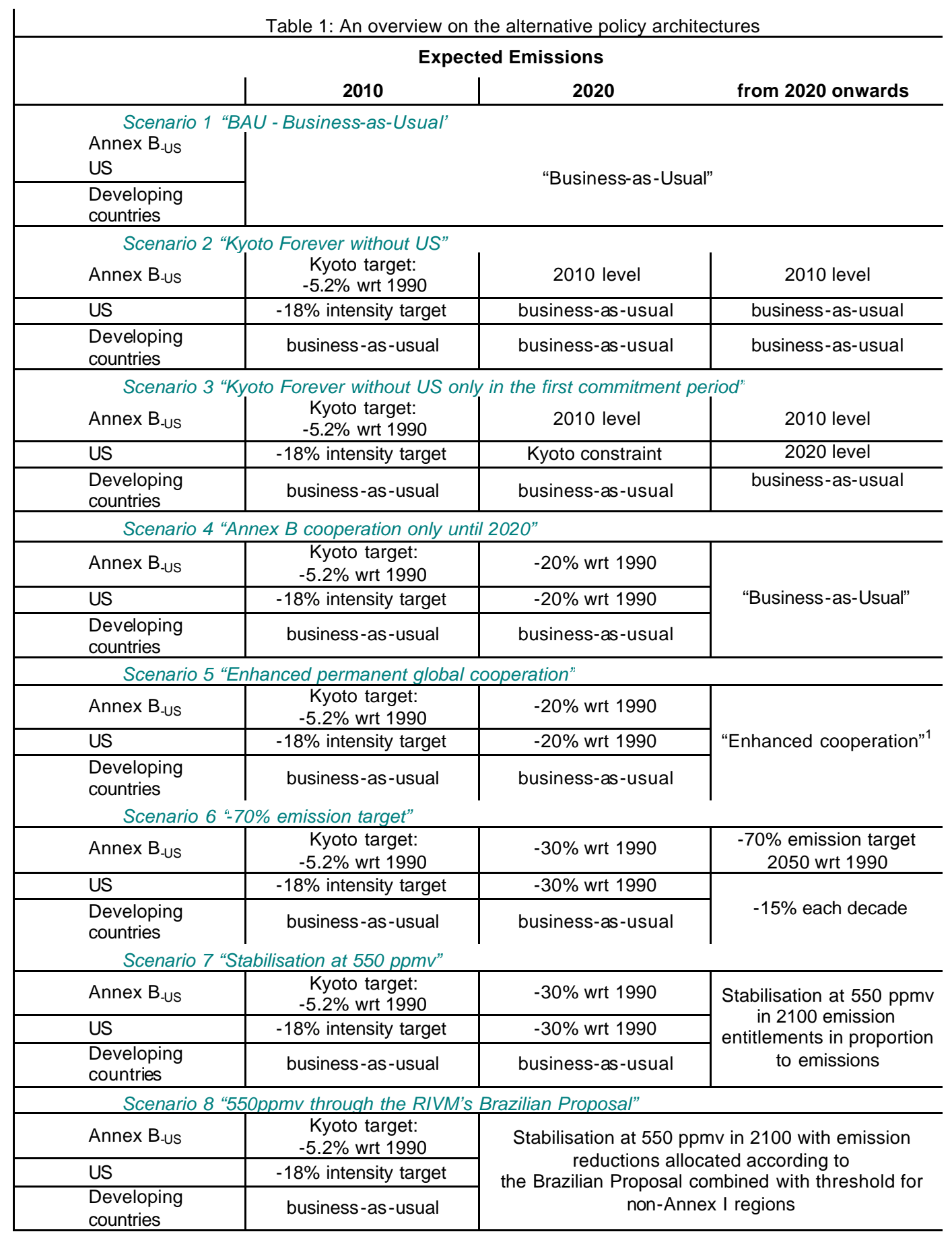




\begin{tabular}{|c|c|c|}
\hline Scenaric & omv through Contract & \multirow{4}{*}{$\begin{array}{l}\text { \& Convergence } \\
\text { Stabilisation at } 550 \text { ppmv in } 2100 \text { with emission } \\
\text { reductions allocated according to } \\
\text { C\&C Approach: All Parties participate immediately in } \\
\text { the climate regime with per capita emission } \\
\text { allowances converging towards equal levels over } \\
\text { time. }\end{array}$} \\
\hline Annex B-us & $\begin{array}{c}\text { Kyoto target: } \\
-5.2 \% \text { wrt } 1990\end{array}$ & \\
\hline US & $-18 \%$ intensity target & \\
\hline $\begin{array}{l}\text { Developing } \\
\text { countries }\end{array}$ & business-as-usual & \\
\hline \multicolumn{3}{|c|}{ Scenario 10 "Stabilisation at 450 ppmv" } \\
\hline Annex $B_{\text {-us }}$ & $\begin{array}{l}\text { Kyoto target: } \\
-5.2 \% \text { wrt } 1990\end{array}$ & \multirow{3}{*}{ Stabilisation at 450 ppmv in 2100} \\
\hline US & $-18 \%$ intensity target & \\
\hline $\begin{array}{l}\text { Developing } \\
\text { countries }\end{array}$ & business-as-usual & \\
\hline
\end{tabular}

\begin{tabular}{|l|c|}
\hline \multicolumn{2}{|c|}{ Table 2: Results of the DEA Ranking of Polcies } \\
\hline \multicolumn{1}{|c|}{ Unit } & Score \\
\hline "Kyoto- US only in 1st commitment period" & 1 \\
\hline "-70\% emission target" & 1 \\
\hline "550ppmv through the RIVM's Brazilian Proposal" & 1 \\
\hline "Enhanced permanent global cooperation" & 0.998646591 \\
"Kyoto- US" & 0.997236684 \\
"Annex B cooperation until 2020" & 0.995300244 \\
"BAU" & 0.995111902 \\
\hline "550ppmv through C\&C" & 0.994655097 \\
\hline "Stabilisation at 550 ppmv" & 0.991907279 \\
\hline "450ppmv through C\&C" & 0.977578886 \\
\hline
\end{tabular}

\begin{tabular}{|c|c|c|c|}
\hline \multicolumn{4}{|c|}{ Table 3: Comparative Advantages of Different Scenarios } \\
\hline Scenario & $\begin{array}{c}C A>0, \text { for } \\
P e>0.5 \text { and } P S>0.5\end{array}$ & $\begin{array}{r}C A>0, \text { for } \\
P e>\varepsilon \text { and } P S>\varepsilon\end{array}$ & $\begin{array}{l}\mathrm{CA}<0 \text {, for } \\
\text { any } \mathrm{Pe} \text { and } \mathrm{PS}\end{array}$ \\
\hline \multicolumn{4}{|l|}{ "BAU" } \\
\hline "Kyoto-US" & & & $\checkmark$ \\
\hline "Kyoto-US only in 1st commitment period" & & $\checkmark$ & $\checkmark$ \\
\hline \multicolumn{4}{|l|}{ "Annex B cooperation until 2020" } \\
\hline "Enhanced permanent global cooperation" & & & $\checkmark$ \\
\hline “-70\% emission target” & $\checkmark$ & $\checkmark$ & $\checkmark$ \\
\hline "Stabilisation at 550 ppmv" & & $\checkmark$ & $\checkmark$ \\
\hline “550ppmv through RIVM's Brazil Proposal” & & $\checkmark$ & $\checkmark$ \\
\hline "550ppmv through C\&C" & & $\checkmark$ & $\checkmark$ \\
\hline "450ppmv through C\&C" & & $\checkmark$ & $\checkmark$ \\
\hline
\end{tabular}

